well enough defined to allow detailed interpretation of function, the results obtained by mutagenesis show clearly the directions in which progress is being made. Those not unduly inhibited by respect for the printed page should be able to keep up with the rapid advance for a year or two by annotation of the many diagrams provided.

Recent developments are well covered, often in separate boxed sections. This avoids disturbance to the general perspective, which is that of the author's earlier book, Transport and Diffusion across Cell Membranes (Academic, 1986), in which he emphasized the physico-chemical approach to understanding transport processes. Many illustrative calculations provide the reader with a feel for how transport systems operate in their cellular milieu. Basic concepts and experimental approaches are clearly explained, so that the general level is suitable for the advanced student, but for the research worker who wants to keep in touch with progress in related fields, there are many tables and diagrams to provide food for thought and an introduction to the appropriate literature. This concise and readable introduction should appeal to a wide audience.

N. Michael Green is at the National Institute for Medical Research, The Ridgeway, Mill Hill, London NW7 1AA, UK.

\section{Astronomy on the box}

\author{
David W. Hughes
}

The Astronomers. By Donald Goldsmith. St Martin's Press: 1991. Pp.332. $\$ 24.95$.

Popularizing science is a Good Thing. The theory is as follows. The more members of the public understand what the scientists are up to, the more excited they become by the quest for discovery and the battle with the unknown. And so the more likely they are to put their hands into their pockets and fund the endeavour, or at least acquiesce to the government putting governmental hands into the pot that contains the communal taxes. To this end science on television is a Very Good Thing because it reaches a huge audience and its intricacies can be explained lavishly and excitingly.

Astronomy has been very lucky in this context. Not only is it replete with beautiful spectacles and ambitious space missions but it also has in its zoo some of the most exotic of creation's creatures. Neutron stars and black holes jostle with big bangs and gravity waves. The subject also has accomplished popularizers such as Patrick Moore and Carl Sagan. In 1980 we were treated, on both sides of the Atlantic, to Sagan's Cosmos, a television series that chronicled the historical develop-

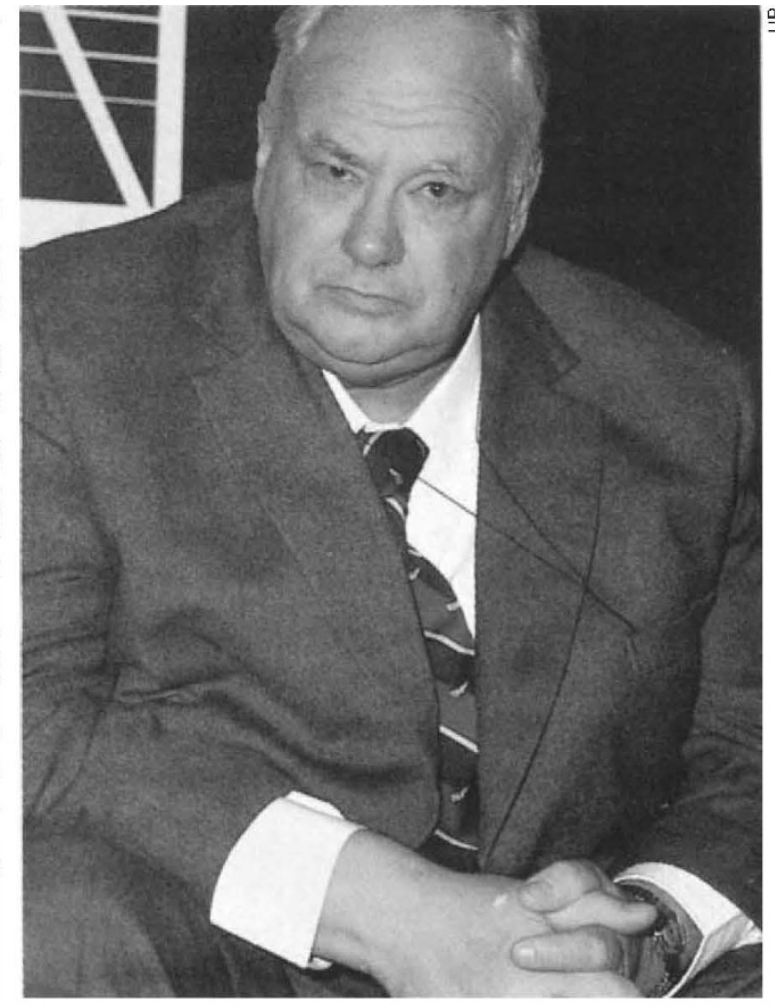

2 Einstein's general theory of relativity is correct, the movement of massive objects should result in the production of gravity waves. Ever more sensitive instruments are being built in the quest for their discovery.

Topic five brings us back to Earth with the investigation of stellar evolution. The book overviews modern theories of the birth of stars and the spectacular deaths of the more massive ones in explosions such as that of Supernova 1987A. Spice is added by an up-to-the-minute account of the search for the pulsar that is one of the last remnants of this astronomical catastrophe.

The last of the six sections stays mainly in our Solar System and relates the drama surrounding the Voyager 2 flyby of Neptune in 1989. Goldsmith also follows some of the more recent astronomical attempts to discover planetary The only astronomer most British TV viewers could name? systems around other stars. Though Patrick Moore often points out that he's not a The Astronomers is lavishly 'professional' scientist.

ment of astronomy. 1991 sees The Astronomers on the US Public Broadcasting Service television stations. The six hour-long episodes introduce the television audience to a supposedly eyewitness perspective of today's professional astronomers beavering away at the cutting edge of their science with state-of-the-art instrumentation and ideas to hand. A sum of $\$ 5.3$ million was awarded by the W. M. Keck Foundation to KCET-TV in Los Angeles, California, for the whole project. Donald Goldsmith, a professional astronomer and former consultant on the Cosmos team wrote the scripts. The book reviewed here is the book of the series.

Now it is rather difficult to divide modern astronomy into just six exciting topics, but I think Goldsmith has chosen well. His first topic concerns missing mass. Astronomers have slowly woken up to the fact that what they see is only a mere fraction of what exists in the Universe. Dark material abounds, and restrains the dissipation of galactic clusters and even makes our own Galaxy much more massive than we previously thought. Unfortunately, we have little idea as to what form this new material takes.

Next we have black holes, and here we rely on long base-line radio interferometry to produce maps of ever-higher resolution of the vicinities of these objects. The third major topic concerns the Big Bang and the detailed investigation of the spectrum and spatial variability of the remnant cosmic background radiation.

When looking for the next great astronomical breakthrough Goldsmith turns to gravitational radiation. If Albert especially was the way in which the procession of 'old favourites', of star fields, planetary surfaces and galactic vistas, was much enlivened by the inclusion of a large number of portraits of living astronomers. The text is rather too gushing. The thumbnail biographical descriptions overflow with complimentary adjectives. Surely all astronomers are not "eager, intelligent, helpful, hard-working, remarkable, renowned, compact, handsome ..."? Goldsmith seems to have moved in exalted circles. Many of the astronomers that I have met cover a much wider spectrum, and suggest adjectives ranging from diffident, uncertain, painstaking and reclusive to bombastic, overbearing, self-opinionated, rude and dismissive.

I also found the title of the book misleading. The Astronomers is really about US astronomers, and few foreigners grace the pages. I am not accusing Goldsmith of being xenophobic but it is quite clear that when he uses the word Cambridge he has in mind a leafy suburb of Boston, Massachusetts, and not an old university town in the English fens. Even when he visited the UK Infrared Telescope on Hawaii he picked one of the infrequent nights when it was being used by two Californians.

In essence, we are presented with an easily digestible popular romp through six of the highlights of the astronomy programme of the United States.

David W. Hughes is in the Department of Physics, University of Sheffield, PO Box 597 , Hicks Building, Hounsfield Road, Sheffield S10 2UN, UK. 